\title{
Beitrag zur Kenntniss des Ectropion uveae congenitum.
}

\author{
Von
}

\begin{abstract}
San--Rath Dr. Bol. Wicherkiewicz in Posen.
Hierzu Fig. 1 und 2 anf Tafel IV.
\end{abstract}

Im Allgemeinen scheinen pigmentartige Excrescenzen am Pupillarrande, wie sie bei Pferden so häufig vorkommen, bei Menschen ziemlich selten beobachtet worden zu sein. In dem vortrefflichen Werk von Wedl und Bock, betitelt: "Pathologische Anatomie des Auges", wird keines entsprechenden Falles Erwähnung gethan; es heisst zwar, solche Excrescenzen seien gewöhnlich wenigstens theilweise pigmenthaltig und führten zu einer vorderen oder hinteren Synechie, oder gar zum Verschluss der Pupille, doch kann damit wohl nicht die congenitale Veränderung, von der ich sprechen will, gemeint sein.

Die Autoren setzen aber hinzu, Holmes (Chicago, med. Journ. 1873) habe bei einem Mediciner am Pupillarrande schwarze schwammig aussehende Massen, wie beim Pferd, die vielleicht angeboren waren, gefunden. Kleinere dunkelbraune Prominenzen am Pupillarrande sonst gesunder Augen wurden $a b$ und zu beobachtet, mögen aber noch offter, da sie sich vom schwarzen Hintergrunde der Pupille nicht abheben, bei gewöhnlicher Tagesbeleuchtung übersehen worden sein.

v. Graefe hat in seinem Arch. für Ophthalm. VII, 2, S. 35 die Beschreibung einer angeborenen Pigmentgeschwulst auf der rechten Iris einer 1ójährigen, gesunden Patientin gegeben. Die Geschwulst von ovaler Gestalt und schwärz- 
Beitrag zur Kenntniss des Ectropion uveae congenitum. 205

lich brauner Farbe, im horizontalen Durchmesser $11 / 2 \mathrm{~mm}$, im verticalen $1 \mathrm{~mm}$ schien aus dem Pupillarrande hervorgegangen zu sein und bewegte sich mit der Iris.

Das Uebel, welches in dieselbe Kategorie von Uvealectropion gehören dürfte, wurde zufälliger Weise von der Patientin bemerkt und v. Graefe beobachtete innerhalb eines Jahres keine Veränderung an der Geschwulst.

Przybylski (Westnik Ophthalm. V, 2, S. 148) beobachtete bei vier Rekruten zottenartige Auswïchse am $\mathrm{Pu}$ pillarrande, und da Anzeichen einer überstandenen Iritis fehlten, so hat er sie als Anomalie des Pigmentsaumes (hyperplastische Wucherung) gedeutet. Przybylski ist mit der von Colsmann vorgeschlagenen Benennung und mit der von anderen Autoren (Hirschberg, Anke, v. Reuss) gebrauchten "Ectropion uveae congenitum" nicht zufrieden und schlägt vor, diese Anomalie "Villositates congenitae strati retinalis marginis pupillaris iridis" zu benennen. Eine etwas lange Bezeichnung, die im Grunde genommen mit dem Ausdruck Villositates uveales marginis pupillaris wohl vertauseht werden dürfte, wenn die Bezeichnung Ectropium uveae congenitum nicht geniigt.

Wenn ich mir nun gestatte kurz über einen derartigen, von mir beobachteten Fall zu berichten, so geschieht dies erstens, weil die Pigmenthervorragungen eine Ausdehnung einnahmen, wie sie man wohl bis dahin bei Menschen nicht beobachtet hat und $z$ weitens, weil ausserdem eine starke Pigmentlage die ganze Hälfte des Vorderstücks der Iris des einen Auges bedeckte und mit den Pigmentexcrescenzen in einiger Beziehung stand. Auch waren noch andere Zeichen einer ausgiebigen Pigmentirung des Auges vorhanden, wie dies aus der nachfolgenden Beschreibung hervorgeht:

Der Arbeiter Joseph H., 44 Jahr alt, erschien am 22. Februar 1889 bei mir, weil seine Kurzsichtigkeit in der letzten Zeit zugenommen habe und seine Augen thränten.

Der ziemlich robuste Mann entstammt einer gesunden Fa- 
milie und hat sieben Geschwister, die alle normale Augen haben sollen.

Die Untersuchming ergiebt für die Ferne $1 / 2$ Sehschärfe bei einer Myopie von 6,5 D., während in der Nähe Jäger 1 gelesen wird. Bei focaler Belenchtung bemerkte ich rechts am unteren Pupillarrande einen, am oberen vier traubenartige $\mathrm{Pu}$ pillarauswüchse, deren Farbe etwas dunkler brann als die der Iris erschien. Am linken Auge sind diese Auswüchse noch zahlreicher und stärker entwickelt und es beträgt die Länge des grössten unter ihnen etwa $3-3,5 \mathrm{~mm}$. Diese Pigmentauswüchse ragen frei in die Vorderkammer, sitzen stellenweise dem Pupillarrande breit auf, stellenweise auf einem schmalen kurzen Stiel und behindern die Bewegung der Pupille gar nicht, auch berühren sie die vordere Linsenfläche nicht, sie sind gefaltet oder höckerig and Trüffehn nicht unähnlich, die stärkeren sind loffelförmig ausgehöhlt, mit der Excavation nach vorne gerichtet.

Nach Atropinisirung haben sich die Pupillen prompt und regelmässig erweitert, die Pigmentanswüchse sind in Folge dessen kürzer aber breiter geworden. Die Linsenkapsel erscheint ganz rein ohne jede Pigmentauflagerung, dagegen zeigt die Linse in der vorderen Corticalis einen zum Linsenrande concentrischen, $8 \mathrm{~mm}$ im Durchmesser messenden und $3 \mathrm{~mm}$ breiten getriubten Ring. Ausserdem bemerkt man namentlich am linken Auge bei Lupenvergrösserung einige feine mohnkorngrosse Trübungen hinter der vorderen Linsenkapsel innerhalb einer mässig erweiterten Pupille. Der Augenhintergrund bot nichts besonderes dar, nur erscheint das Chorioidealpigment stark entwickeit. An diesem Auge bemerikte man ausserdem noch andere Abweichungen: über der Hornhant nach oben und innen etwa $3 \mathrm{~mm}$ vom Hornhautrande entfernt, fanden sich zwei mit einander durch eine Brücke verbundene hanflorngrosse Pigmenteinlagerungen in der Sclera und weiter oben mehrere solche, aber nur punktförmige; die vordere Irisfläche ist in ihrer oberen inneren Hälfte von einer Pigmentlage bedeckt, die jener der Auswüchse an Farbe ganz gleich kommt, mit ihnen unmittelbar verbunden erscheint und durch die Ausfüllung der Vertiefungen zwischen den Leisten dem Irisgewebe im entsprechenden Theile der Regenbogenhaut ein glattes sammetartiges Aussehen verleihen. Auch sieht man innerhalb der äusseren unteren vom Uvealblatte nicht bedeckten Irishälfte eine etwa hanfkorngrosse Pigmentinsel. 
Beitrag zur Kenntniss des Ectropion uveae congenitum. 207

Unzweifelhaft ist die beschriebene Anomalie congenitalen Ursprungs. Der Träger derselben hatte davon keine Ahnung und keine Störung irgend welcher Art. Das, was ihn zu mir führte, war die Kurzsichtigkeit und vielleicht die geringe Linsentrübung, von der bis dahin nicht festgestellt werden konnte, ob sie zunimmt oder stationär ist.

Als Product einer Entzündung können die Excrescenzen nicht aufgefasst werden, da erstens die Iris sonst ein vollkommen normales Aussehen hat, und ferner absolut keine Pigmentauflagerungen auf der Linsenkapsel, wie sie sonst wohl vorkommen würden, und auch unter anderen von Bock (Klin. Monatsbl. 1888, April, S. 168) nachgewiesen wurden, bei minutiösester Untersuchung festgestellt werden konnten.

Die Frage, auf welche Weise diese Excrescenzen zu Stande gekommen, lässt sich mit positiver Sicherheit nicht beantworten, doch hat die von Bock vertretene Ansicht, dass es sich hier um eine mangelhafte Involution der Pupillarmembran handele, viel bestechendes für sich, um so mehr als in den zur histologischen Untersuchung gekommenen Fällen nicht nur reines Uvealpigment, sondern auch pigmentirtes Bindegewebe oder ein atrophischem Irisgewebe ähnliches Stroma mit Pigment gefunden wurde (Businelli). Immerhin wird man eine besonders starke fötale Hyperplasie des Uvealpigments annehmen müssen, wofür nicht nur jene Excrescenzen, sondern auch die auf dem linken Auge in unserem Falle sichtbare Pigmentauflagerung auf der Irisfläche und Einlagerung auf der Sclera zu sprechen scheinen.

Die pigmentirten Excrescenzen können sich nach neueren Erfahrungen (Bock, Fuchs, Kummer, Streatfield, Schneider, Troicki) vom Pupillarrande loslösen und als Fremdkörper in der Vorderkammer suspendirt bleiben, und auch gelegentlich zur Bildung von Cysten wie im Fall Fuchs (Archiv für Augenheilk. XV, S. 7) führen. 